\title{
High Dose of Botulinum Toxin Type A (NT-201) for the Treatment of Dysphagia due to Severe Spasticity of Upper Oesophageal Sphincter: A Case Report
}

\section{Basciani and D Intiso*}

Unit of Neuro-Rehabilitation, IRCCS “Casa Sollievo della Sofferenza” Hospital, San Giovanni Rotondo (FG), Italy

\begin{abstract}
Background: Botulinum toxin type A (BoNT-A) has been used in treating dysphagia due to spasticity of upper oesophageal sphincter (UES). However, the doses commonly injected could result ineffective. A case of dysphagia treated successfully by high dosage of BoNT-A is described.

Case report: A50 year-old male subject with tetraparesis and dysphagia due to severe spasticity of UES following encephalitis. No bolus transit was observed to videofluoroscopy (VS). Because of severe UES spasticity, it was not possible to perform oesophageal manometry. Growing BoNT-A dosage of $10 \mathrm{U}$ (Botox) and $100 \mathrm{U}$ (Xeomin) injected into cricopharingeal muscle (CM) had no benefit. Since negative effect of previous doses, BoNT-A (Xeomin) high dosage of $200 \mathrm{U}$ was injected into CM. After neurotoxin injections,VS showed significant dysphagia improvement lasting eight months. Transient paresis of the right vocal cord occurred. A second BoNT-A injection session with same dosage and modalities was performed efficaciously.
\end{abstract}

Conclusion: High dose of $200 \mathrm{U}$ of BoNT-A (Xeomin) was efficacious and safe in treating refractory dysphagia due to severe spasticity of UES. High dosage of neurotoxin should be considered when common doses were ineffective.

Keywords: Dysphagia; Botulinum toxin; Spasticity; Encephalitis

\section{Introduction}

Dysphagia is a dysfunction in normal swallowing characterized by a condition that the food including solids or liquids are not being swallowed correctly. Swallowing is a highly coordinated physiologic event that requires sequential and overlapping movements of the facial, oral, pharyngeal, laryngeal and esophageal muscular apparatus, all culminating in transit of the ingested material and oral secretions from the mouth into the stomach [1]. Oral, pharyngeal, and oesophageal phase characterize the normal swallowing, all of which can be affected resulting in dysphagia. Hence, it can follow a huge of disturbances including oropharyngeal, neurological diseases, and postsurgical disorders. One of the most important structures involved in normal swallowing is the Upper Esophageal Sphincter (UES) which is predominantly regulated by Cricopharyngeus Muscle (CM) action. Among the disorders of swallowing, oropharyngeal dysphagia due to hyperactivity of the CM is the prevalent abnormality. The structure of the CM maintains constant luminal occlusion at rest, but allows relaxation and contraction during swallowing [2]. In treating the dysphagia due to CM hyperactivity, several treatments including mechanical dilatation, pharyngeal plexus neurectomy [3], and surgical myotomy [4] have been proposed with variable benefit. Myotomy of CM is the treatment of choice for the hyperactivity of the UES due to neurologic disorders [5], but it is generally suggested when other interventions have failed. BoNT-A has been widely reported as efficacious therapeutic medical tool for the treatment of dysphagia due to the spasticity of CM [6-8]. However, when using BoNT-A for the treatment of dysphagia, the doses that are commonly injected could result ineffective. The available BoNT-A agents are abobotulinumtoxinA, onabotulinumtoxinA and incobotulinumtoxinA, commercially named Dysport, Botox and Xeomin, respectively. The use of incobotulinumtoxinA high dose in a case of resistant dysphagia due to severe spasm of the UES is reported.

\section{Case Report}

A 50-year-old male patient with clinical history of bacterial encephalitis contracted two years previously. Clinical and neurological pictures were characterized by spastic tetraparesis and severe dysphagia requiring tube feeding. Repeated Video Fluoroscopy (VS) showed dysfunction of the UES due to severe spasticity of $\mathrm{CM}$ that was confirmed by EMG evaluation. Oral and pharyngeal phases of swallowing were retained. Notwithstanding rehabilitation and pneumatic oesophageal dilatation, the dysphagia unchanged and the subject needed of percutaneous endoscopic gastrostomy. In order to improve swallowing, the patient underwent BoNT-A injection of spastic CM. Initially, a percutaneous dose of $10 \mathrm{U}$ of onabotulinumtoxinA was injected in one site of CM by EMG guide using a teflon-coated needle, unsuccessfully. Four months later, $100 \mathrm{U}$ of incobotulinutoxinA diluted at $25 \mathrm{U} / \mathrm{ml}$ were injected in 3 sites of $\mathrm{CM}$ by endoscopic guidance. Each point was injected by 30,30 and 40 $\mathrm{U}$ of BoNT-A, but the dysphagia unchanged. Since BoNT-A resulted ineffective a surgical option consisting of CM myotomy was suggested, but the patient firmly refused the surgical intervention. The subject developed anxiety, severe mood depression and complained a scanty quality of life. Because repeated and imploring requests to improve the swallowing symptoms and to gain oral nutrition, a further neurotoxin treatment by using higher dosage was proposed. After the patients had given written informed consent, $200 \mathrm{U}$ of incobotulinumtoxinA were injected into spastic UES by endoscopic guidance. BoNT-A was diluted with $0.9 \mathrm{ml}$ sodium chloride solution at $25 \mathrm{U} / \mathrm{ml}$, but unlike previous

*Corresponding author: Domenico Intiso, Unit of Neuro-Rehabilitation IRCCS "Casa Sollievo della Sofferenza", Italy, Tel: 039882410 942; E-mail: d.intiso@ operapadrepio.it

Received August 08, 2015; Accepted August 18, 2015; Published August 25, 2015

Citation: Basciani M, Intiso D (2015) High Dose of Botulinum Toxin Type A (NT-201) for the Treatment of Dysphagia due to Severe Spasticity of Upper Oesophageal Sphincter: A Case Report. J Clin Case Rep 5: 577. doi:10.4172/2165-7920.1000577

Copyright: ( 2015 Basciani M, et al. This is an open-access article distributed under the terms of the Creative Commons Attribution License, which permits unrestricted use, distribution, and reproduction in any medium, provided the original author and source are credited. 
two treatments, the neurotoxin was injected in 3 equidistant points along two perimeters of $\mathrm{CM}$, distant one $\mathrm{cm}$ from each other. Each site received 30, 30 and $40 \mathrm{U}$ of BoNT-A, respectively. VS with modified barium swallowing, and oesophageal manometry were used to assess the dysphagia before and 2 weeks after BoNT-A injections. Objective swallowing evaluation was also ascertained by Penetration-Aspiration Scale (PAS) described elsewhere [9]. The scale ranges from 1 (no penetration or aspiration) to 8 (aspiration without cough response). Before BoNT-A treatment, VS showed no passage of barium into the oesophagus with occasional pharyngeal reflux due to severe UES spasticity (Figure 1). Poor contractility of the lower pharyngeal segment with massive movement was observed, but no aspiration occurred. Oesophageal manometry was not performed, because severe spasm of the UES did not permit probe transit. PAS value scores were 1 and 0 for liquid and solids, respectively. After two weeks from BoNTA injection, VS showed significant improvement of swallowing consisting of good bolus transit into the oesophagus, even if the transit was slightly delayed (Figure 2). Normal values were detected to oesophageal manometry. Likewise, improvement of PAS scale was detected: 7 and 6 for liquid and solid, respectively. The subject complained only mild pain in the

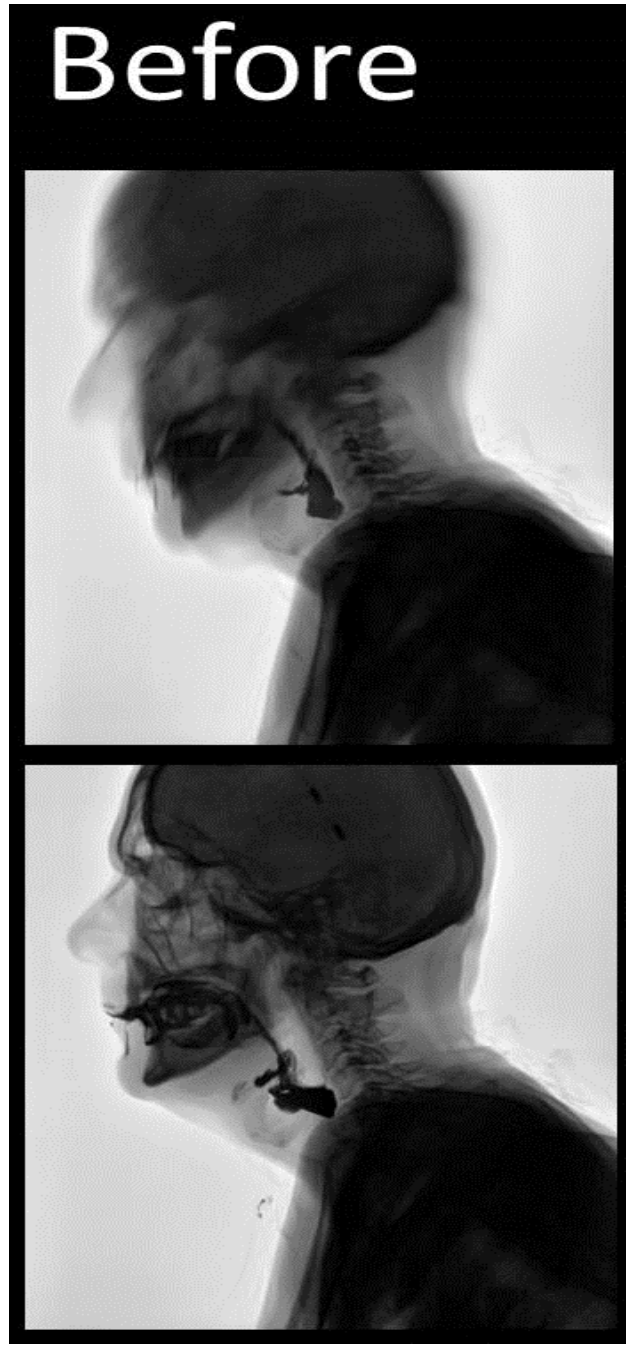

Figure 1: VS before BoNT-A: complete absence of the barium transit into oesophagus due to UES severe spasticity. Oral and pharyngeal phases of swallowing are maintained.

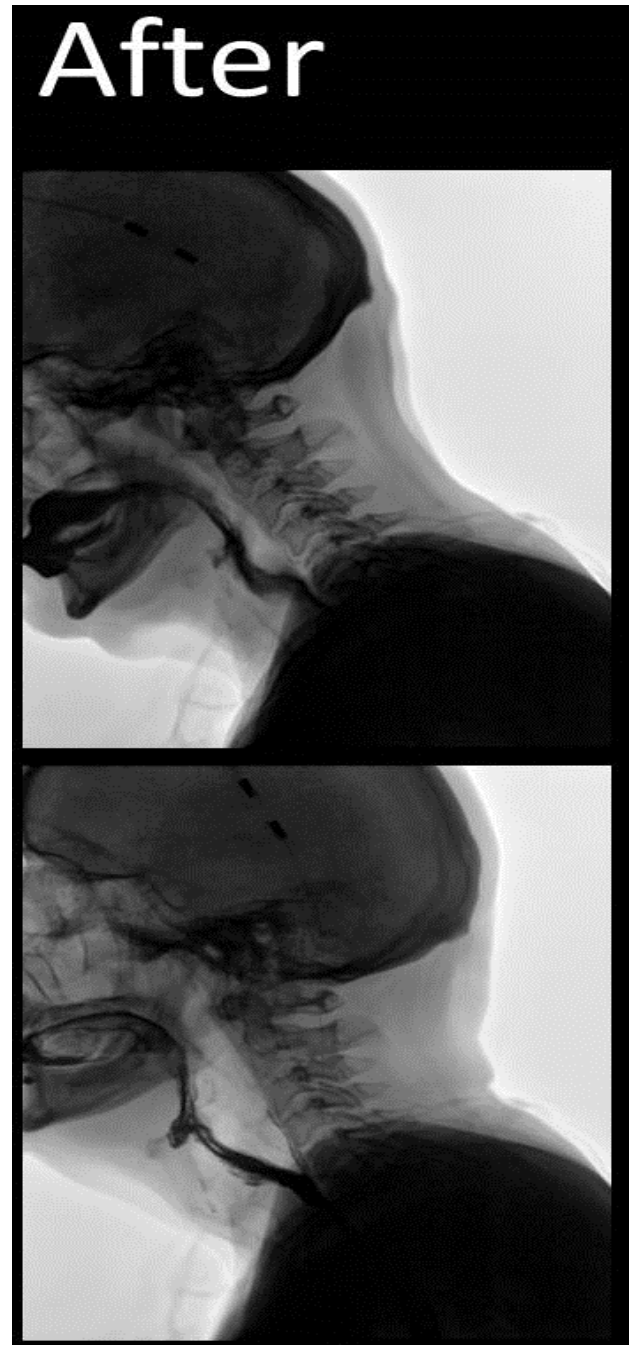

Figure 2: VS after 2 weeks from BoNT-A: passage of the barium through the oesophagus. Mild barium stasis in pyriform sinus is present

site of the neurotoxin inoculation and showed paresis in abduction of the right vocal cord lasting two weeks. On the other hand, he gained almost complete oral feeding that persisted for nearly eight months. At this time, a second BoNT-A injection session with same dosage and modalities was performed that maintained the swallowing benefit previously obtained. No side effect was observed, apart mild pain in the site of inoculation.

\section{Discussion}

High-dose of $200 \mathrm{U}$ of incobotulinumtoxinA injected into CM was effective in reducing persistent dysphagia due to severe spasticity of the UES in an adult subject with encephalitis. The treatment produced long-term swallowing benefit with mild side effect consisting of temporary right vocal cord paresis.

Botulinum toxin has been widely reported as efficacious therapeutic medical tool for the treatment of dysphagia due to spasticity of CM. Although BoNT-A could represent an alternative therapeutic strategy to the surgical option, the dysphagia could be unchanged by neurotoxin treatment. Zaninotto et al. [10] reported that BoNT-A 
was effective in only $40 \%$ of subjects with dysphagia following various diseases [10]. Several reasons could explain negative results as such neurotoxin formulation, dilutions, variability of points of injections from single or multiple shots, and variability in the delivery techniques. All aspects suggesting that neurotoxin dosage may not reach $\mathrm{CM}$, consistently. Furthermore, BoNT-A effect could be due to the injected dose. Indeed, the quantity of BoNT-A injected varies considerably, ranging from 2.5 to $120 \mathrm{U}$ for onabotulinumtoxin $\mathrm{A}$, and from 60 to $300 \mathrm{U}$ for abobotulinumtoxinA [7]. So far, the administrated doses have not exceeded $120 \mathrm{U}$ for onabotulinumtoxinA and $360 \mathrm{U}$ for abobotulinumtoxinA [7]. To our knowledge, this is the first report concerning the use of BoNT-A high dose in reducing dysphagia due to severe CM spasticity, as well the first description in the use of incobotulinumtoxinA for such indication. IncobotulinumtoxinA has similar biologic activity to onabotulinumtoxinA [11] and its potency is suggested to be equivalent. Recently, high doses of incobotulinumtoxinA have been demonstrated effective and safe for the treatment of spasticity due to stroke, brain injury and cerebral palsy [12]. In the present case, a high dose of $200 \mathrm{U}$ of incobotulinumtoxin $\mathrm{A}$ was injected because the dosages commonly used in treating spastic UES were ineffective and the patients firmly refused cricopharingeal myotomy. It is possible that not all patients suffering from severe dysphagia could have benefit by high BoNT-A dosage. Zaninotto et al. [10] suggested that the best candidates to BoNT-A treatment are the subjects showing less severely damaged swallowing function at VS and CP spasm still capable of propelling the bolus through the mouth and pharynx [10]. Anyway, in treating dysphagia by BoNT-A agent, whenever common doses fail, we suggest to use high dosage of neurotoxin before proposing other therapeutic strategies. The major concern of using BoNT-A at a high dosage is the risk of remote adverse events consisting of excessive weakness and botulism and resistance to BoNT-A for neutralizing antibodies production. Dressler reported that incobotulinumtoxin A and onabotulinumtoxin A formulations can be used in doses of up to $840 \mathrm{U}$ without producing clinically detectable systemic adverse events [11]. Indeed, the patient of present case complained only mild pain in the site of the neurotoxin inoculation and developed paresis in abduction of the right vocal cord lasting two weeks. On the other hand, incobotulinumtoxinA high dose produced benefit of swallowing for about eight months.

\section{Conclusion}

High dosage of $200 \mathrm{U}$ of incobotulinumtoxinA was effective and safe in treating dysphagia due to severe spasticity of UES that was resistant to common BoNT-A doses. The use of high BoNT-A dosage should be considered before suggesting surgical procedures, whenever the common therapeutic doses resulted ineffective. Written informed consent was obtained from the patient for publication of this case report and accompanying images.

\section{References}

1. Restivo DA, Casabona A, Nicotra A, Zappia M, Elia M, et al. (2013) ALS dysphagia pathophysiology: differential botulinum toxin response. Neurology 80: 616-620.

2. Haapaniemi JJ, Laurikainen EA, Pulkkinen J, Marttila RJ (2001) Botulinum toxin in the treatment of cricopharyngeal dysphagia. Dysphagia 16: 171-175.

3. Singer MI, Blom ED, Hamaker RC (1986) Pharyngeal plexus neurectomy for a laryngeal speech rehabilitation. Laryngoscope 96: 50-54.

4. Woodson GA (1997) Cricopharyngeal myotomy and arytenoids adduction in the management of combined laryngeal and pharyngeal paralysis. Otolaryngo Head Neck Surg 116: 339 -343.

5. Born LJ, Harned RH, Rikkers LF, Pfeiffer RF, Quigley EM (1996) Cricopharyngeal dysfunction in Parkinson's disease: role in dysphagia and response to myotomy Mov Disord 11: 53-58.

6. Kelly EA, Koszewski IJ, Jaradeh SS, Merati AL, Blumin JH, et al. (2013) Botulinum toxin injection for the treatment of upper esophageal sphincter dysfunction. Ann Otol Rhinol Laryngol 12: 100-108.

7. Moerman MB (2006) Cricopharyngeal Botox injection: indications and technique. Curr Opin Otolaryngol Head Neck Surg 14: 431-436.

8. Woisard-Bassols V, Alshehri S, Simonetta-Moreau M (2013) The effects of botulinum toxin injections into the cricopharyngeus muscle of patients with cricopharyngeus dysfunction associated with pharyngo-laryngeal weakness. Eur Arch Otorhinolaryngology 270: 805-815.

9. Rosenbek JC, Robbins JA, Poeckeer EB (1996) A penetration aspiration scale. Dysphagia 11: 93 -98.

10. Zaninotto G, Marchese Ragona R, Briani C, Costantini M, Rizzetto C, et al. (2004) The role of botulinum toxin injection and upper esophageal sphincter myotomy in treating oropharyngeal dysphagia. J Gastrointest Surg 8: 997-1006.

11. Dressler D (2008) Equivalent potency of Xeomin $®$ and Botox $®$. MovDisord 23 20-21.

12. Intiso D, Simone V, Di Rienzo F, larossi A, Pazienza L, et al. (2014) High doses of a new botulinum toxin type A (NT-201) in adult patients with severe spasticity following brain injury and cerebral palsy. NeuroRehabilitation 34: 515-22. 